\title{
Global stability in a model of the glucose-insulin interaction with time delay
}

\author{
D. L. BENNETT and S. A. GOURLEY \\ Department of Mathematics and Statistics, University of Surrey, Guildford, Surrey GU2 7XH, UK \\ email: s.gourley@surrey.ac.uk
}

(Received 18 August 2002; revised 10 June 2003)

\begin{abstract}
A variety of models on the interaction between glucose and insulin have been suggested over the last 50 years. One, developed by Sturis et al. [19], and consisting of six nonlinear ordinary differential equations, has been widely accepted. However, the model has the disadvantage of containing auxiliary variables which have no clinical interpretation. In this paper we study an alternative model which incorporates a time delay explicitly, negating the need for the auxiliary equations. A simplifying assumption of having just one insulin compartment reduces the number of equations still further. We then study the resulting system of two differential delay equations, establishing results on positivity, boundedness, persistence and global asymptotic stability. For the latter, two quite different approaches are employed: comparison principles and Lyapunov functionals. The two approaches provide different sets of sufficient conditions for global stability, so that we investigate different regions of parameter space.
\end{abstract}

\section{Introduction}

Over the last 50 years the interaction between glucose and insulin, its regulatory hormone, has been studied by both theoretical and mathematical biologists [1, 5, 8]. Through biological experiments it has been well-established $[6,9,17,18]$ that insulin secretion in the pancreas oscillates on a number of different time scales, ranging from tens of seconds to more than 100 minutes. The oscillations with larger period (80-150 mins) are known as ultradian oscillations and a model developed by Sturis et al. [19] (see also Keener $\&$ Sneyd [7]) provides a possible mechanism for their origin. This model consists of six nonlinear ordinary differential equations and is detailed in Appendix I (system (4.1)).

Whilst Sturis' model (recently modified by Tolic et al. [20] to contain a more sophisticated receptor down-regulation model and receptor modification model) is consistent with observable features of ultradian insulin oscillations, it has the disadvantage of artificially introducing auxiliary variables which have no clinical interpretation. In this article we introduce time delay into the model explicitly, thereby negating the need for the three auxiliary linear chain equations and their associated artificial parameters. In addition, we make the further simplifying assumption that plasma and intercellular insulin are indistinguishable. The original model is thus reduced from six ODEs without delay, to two equations with delay. Li et al. [10] proposed a delay model which has certain similarities to the model we propose in this paper, but their model has the delay in the insulin equation. 
The model of the present paper was also considered by Engelborghs et al. [3]. In fact, they modified it to represent an external system interacting with an internal system in the case of a diabetic patient. They studied the linearised stability of the equilibria and carried out some numerical bifurcation analysis. They extensively investigated several branches of periodic solutions and their stability.

In the present paper, we study the simplified system analytically to determine some of its fundamental properties and, especially, to obtain theorems on the global stability of the equilibria. Also, we have aimed to keep the functions $f_{i}$ (in system (2.1) below) as general as possible, rather than restricting to the particular $f_{i}$ mentioned in Appendix A. We make only general qualitative assumptions on the $f_{i}$; those that are dictated by the need for biological realism. The paper is organised as follows: in $\S 2$ we present our model and some of its basic properties, $\S 3$ addresses global stability, Appendix A summarises the model of Sturis et al. [19] and Appendix B lists the properties of the functions $f_{i}$ used in our model.

\section{The model equations and preliminary results}

We first modify Sturis' model by explicitly incorporating a discrete delay term into the glucose equation. In this way the three auxiliary variables of Sturis's model representing the delay between plasma insulin and its effect on hepatic glucose production can be dispensed with. To reduce the number of equations still further, we assume there is only one insulin compartment rather than two (i.e. no distinction between plasma insulin and intracellular insulin). Therefore, $t_{p}$ and $t_{i}$ in system (4.1) are taken to be equal and we introduce $I=I_{p}+I_{i}$. These modifications yield the following model to be solved for $t>0$ :

$$
\begin{gathered}
d I / d t=f_{1}(G)-\frac{1}{\tau_{0}} I, \\
d G / d t=G_{i n}-f_{2}(G)-q G f_{4}(I)+f_{5}(I(t-\tau)), \\
I(s)=I_{0}(s) \geqslant 0, \quad s \in[-\tau, 0] \quad \text { with } I_{0}(0)>0, \\
G(0)=G_{0}>0,
\end{gathered}
$$

where the functions $f_{1}, f_{2}, f_{4}$ and $f_{5}$ satisfy the assumptions in Appendix B, and $q>0$ is a constant. Note that there is no function labelled $f_{3}$. In fact, $f_{3}(G)$ is the linear term $q G$ in the second equation. Since Sturis et al. [19] took their function $f_{3}(G)$ to be linear (see the third equation of system (4.1) in Appendix A, and the expression (4.5) for their $f_{3}(G)$ ), it seemed more convenient for us to take $f_{3}(G)$ as $q G$ at the outset, while keeping the other $f_{i}$ general. We decided to retain the original subscripts on the functions $f_{4}$ and $f_{5}$ to allow direct comparison with the original paper.

In (2.1), $I$ and $G$ represent the quantities of insulin (mU) and glucose (mg), respectively. Pancreatic insulin production controlled by glucose concentration is represented by the function $f_{1}(G) . I / \tau_{0}$ is the degradation rate of insulin by the body and $G_{\text {in }}>0$ represents the input of glucose from outside the system. Glucose uptake by the brain and nerve cells is described by the function $f_{2}(G)$. Glucose utilization by muscle and fat cells which is dependent on both glucose and insulin concentration is represented by the third term in the second equation of (2.1). The last term in the second equation of (2.1) represents hepatic glucose production which is influenced by insulin. 


\subsection{Positivity and boundedness}

Proposition 2.1 Let the $f_{i}$ satisfy the assumptions listed in Appendix B. Then all solutions of the model (2.1) exist for all $t>0$ and are strictly positive.

Proof Let $(G(t), I(t))$ be a solution of (2.1). If $G\left(t_{0}\right)=0$ for some $t_{0}>0$, and if $t_{0}$ is the first such time, then $\dot{G}\left(t_{0}\right) \leqslant 0$. However, at $t_{0}$, the glucose equation becomes

$$
\dot{G}\left(t_{0}\right)=\underbrace{G_{i n}}_{>0}-\underbrace{f_{2}\left(G\left(t_{0}\right)\right)}_{=0}-\underbrace{q G\left(t_{0}\right)}_{=0} f_{4}\left(I\left(t_{0}\right)\right)+\underbrace{f_{5}\left(I\left(t_{0}-\tau\right)\right)}_{>0}>0 .
$$

This is a contradiction. Therefore, $G(t)>0$ for all $t>0$. By similar reasoning, $I(t)>0$ for all $t$.

Proposition 2.2 Let the $f_{i}$ satisfy the assumptions listed in Appendix B. Then all solutions of the model (2.1) are bounded.

Proof First we establish the boundedness of $I(t)$. Solving the first equation of (2.1) for $I(t)$ we have

$$
I(t)=e^{\frac{-t}{\tau_{0}}} I(0)+e^{\frac{-t}{\tau_{0}}} \int_{0}^{t} e^{\frac{s}{\tau_{0}}} \underbrace{f_{1}(G(s))}_{\leqslant f_{1}(\infty)} d s \leqslant e^{\frac{-t}{\tau_{0}}} I(0)+f_{1}(\infty) \tau_{0}\left(1-e^{\frac{-t}{\tau_{0}}}\right)
$$

and thus $I(t)$ is bounded for all $t$.

From the second equation of (2.1) we have

$$
\begin{aligned}
\dot{G}(t) & =G_{i n}-f_{2}(G)-q G f_{4}(I)+f_{5}(I(t-\tau)) \leqslant G_{i n}-q \underbrace{f_{4}(I)}_{\geqslant f_{4}(0)}+f_{5}(I(t-\tau)) \\
& \leqslant G_{i n}-q G f_{4}(0)+\underbrace{f_{5}(I(t-\tau))}_{\leqslant f_{5}(0)} \leqslant G_{i n}-q G f_{4}(0)+f_{5}(0) .
\end{aligned}
$$

Therefore,

$$
\limsup _{t \rightarrow \infty} G(t) \leqslant \frac{G_{i n}+f_{5}(0)}{q f_{4}(0)}
$$

and also

$$
G(t) \leqslant M_{G}:=\max \left\{G(0), \frac{1}{q f_{4}(0)}\left(G_{i n}+f_{5}(0)\right)\right\}
$$

for all $t$. The proof is complete.

Let $(G(t), I(t))$ be a solution of (2.1). Throughout this paper, we define

$$
\bar{G}=\limsup _{t \rightarrow \infty} G(t), \underline{G}=\liminf _{t \rightarrow \infty} G(t), \bar{I}=\limsup _{t \rightarrow \infty} I(t), \underline{I}=\liminf _{t \rightarrow \infty} I(t) .
$$

By Propositions 2.1 and 2.2, these quantities are all finite. The following well known fluctuation lemma is stated below without proof: 
Lemma 2.3 Let $f: \mathbf{R} \rightarrow \mathbf{R}$ be a differentiable function. If

$$
l=\liminf _{t \rightarrow \infty} f(t)<\limsup _{t \rightarrow \infty} f(t)=L,
$$

then there are sequences $\left\{t_{k}\right\} \uparrow \infty,\left\{s_{k}\right\} \uparrow \infty$ such that, for all $k$,

$$
f^{\prime}\left(t_{k}\right)=f^{\prime}\left(s_{k}\right)=0, \lim _{k \rightarrow \infty} f\left(t_{k}\right)=L \text { and } \lim _{k \rightarrow \infty} f\left(s_{k}\right)=l .
$$

Proposition 2.4 Model (2.1) is uniformly persistent, i.e. solutions are eventually uniformly bounded from above and below.

Proof If $\underline{I}<\bar{I}$ then, by Lemma 2.3, there exist sequences $\left\{t_{k}\right\} \uparrow \infty,\left\{s_{k}\right\} \uparrow \infty$, such that

$$
\dot{I}\left(t_{k}\right)=\dot{I}\left(s_{k}\right)=0, \quad \lim _{k \rightarrow \infty} I\left(t_{k}\right)=\bar{I} \quad \text { and } \quad \lim _{k \rightarrow \infty} I\left(s_{k}\right)=\underline{I} .
$$

Thus, from the first equation of (2.1), we have

$$
0=\dot{I}\left(t_{k}\right)=f_{1}\left(G\left(t_{k}\right)\right)-\frac{1}{\tau_{0}} I\left(t_{k}\right)
$$

and

$$
0=\dot{I}\left(s_{k}\right)=f_{1}\left(G\left(s_{k}\right)\right)-\frac{1}{\tau_{0}} I\left(s_{k}\right)
$$

for all $k$.

Let $\varepsilon>0$ be arbitrary. Then there exists $T_{0}>0$ such that, for all $t \geqslant T_{0}$,

$$
G(t) \leqslant \bar{G}+\varepsilon
$$

Also, there exists an integer $k_{0}$ such that $k \geqslant k_{0} \Rightarrow t_{k} \geqslant T_{0}$ and, therefore,

$$
G\left(t_{k}\right) \leqslant \bar{G}+\varepsilon
$$

Hence, for $k$ sufficiently large,

$$
0=f_{1}\left(G\left(t_{k}\right)\right)-\frac{1}{\tau_{0}} I\left(t_{k}\right) \leqslant f_{1}(\bar{G}+\varepsilon)-\frac{1}{\tau_{0}} I\left(t_{k}\right)
$$

since $f_{1}$ is increasing. Letting $k \rightarrow \infty$ and then $\varepsilon \rightarrow 0$,

$$
\bar{I} \leqslant \tau_{0} f_{1}(\bar{G})
$$

In a similar way, we can show using the sequence $s_{k}$ that

$$
\underline{I} \geqslant \tau_{0} f_{1}(\underline{G}) .
$$

Combining (2.2) and (2.3),

$$
\tau_{0} f_{1}(\underline{G}) \leqslant \underline{I}<\bar{I} \leqslant \tau_{0} f_{1}(\bar{G}) .
$$


Recall from the proof of Proposition 2.2 that

$$
\bar{G} \leqslant \frac{1}{q f_{4}(0)}\left(G_{i n}+f_{5}(0)\right) .
$$

so that $G$ is bounded above. Note that (2.4) recovers the result that $I$ is bounded above also. We now need to prove that $\underline{G}>0$ and $\underline{I}>0$.

If $\underline{G}<\bar{G}$ then there exist sequences $\left\{t_{k}^{\prime}\right\} \uparrow \infty,\left\{s_{k}^{\prime}\right\} \uparrow \infty$, such that

$$
\dot{G}\left(t_{k}^{\prime}\right)=\dot{G}\left(s_{k}^{\prime}\right)=0, \quad \lim _{k \rightarrow \infty} G\left(t_{k}^{\prime}\right)=\bar{G} \quad \text { and } \quad \lim _{k \rightarrow \infty} G\left(s_{k}^{\prime}\right)=\underline{G} .
$$

The second equation of (2.1) then gives, for all $k$,

$$
0=\dot{G}\left(s_{k}^{\prime}\right)=G_{i n}-f_{2}\left(G\left(s_{k}^{\prime}\right)\right)-q G\left(s_{k}^{\prime}\right) f_{4}\left(I\left(s_{k}^{\prime}\right)\right)+f_{5}\left(I\left(s_{k}^{\prime}-\tau\right)\right) .
$$

Let $\varepsilon>0$. Then there exists $T_{2}>0$ such that, for all $t \geqslant T_{2}, I(t) \leqslant \bar{I}+\varepsilon$. For all $k$ sufficiently large, $s_{k}^{\prime}-\tau \geqslant T_{2}$ and therefore $I\left(s_{k}^{\prime}-\tau\right) \leqslant \bar{I}+\varepsilon$. Hence, for $k$ sufficiently large,

$$
\begin{aligned}
0 & =G_{i n}-f_{2}\left(G\left(s_{k}^{\prime}\right)\right)-q G\left(s_{k}^{\prime}\right) f_{4}\left(I\left(s_{k}^{\prime}\right)\right)+f_{5}\left(I\left(s_{k}^{\prime}-\tau\right)\right) \\
& \geqslant G_{i n}-f_{2}\left(G\left(s_{k}^{\prime}\right)\right)-q G\left(s_{k}^{\prime}\right) f_{4}(\bar{I}+\varepsilon)+f_{5}(\bar{I}+\varepsilon)
\end{aligned}
$$

since $f_{4}$ is increasing and $f_{5}$ decreasing. Letting $k \rightarrow \infty$ and then $\varepsilon \rightarrow 0$,

$$
0 \geqslant G_{\text {in }}-f_{2}(\underline{G})-q \underline{G} f_{4}(\bar{I})+f_{5}(\bar{I}) .
$$

Now suppose, for contradiction, that $\underline{G}=0$. Substituting this into (2.6) gives

$$
0 \geqslant G_{\text {in }}+f_{5}(\bar{I})>0
$$

a contradiction. Therefore $\underline{G}>0$. From (2.4) we now have $\underline{I}>0$ also. The proof is complete.

\subsection{Equilibria}

Let us investigate the equilibria $\left(I^{*}, G^{*}\right)$ of our system. The first equation of (2.1) gives

$$
I^{*}=\tau_{0} f_{1}\left(G^{*}\right)
$$

From this, we obtain a single equation for $G^{*}$ :

$$
0=G_{i n}-f_{2}\left(G^{*}\right)-q G^{*} f_{4}\left(\tau_{0} f_{1}\left(G^{*}\right)\right)+f_{5}\left(\tau_{0} f_{1}\left(G^{*}\right)\right)
$$

Define

$$
h(G)=G_{i n}-f_{2}(G)-q G f_{4}\left(\tau_{0} f_{1}(G)\right)+f_{5}\left(\tau_{0} f_{1}(G)\right) .
$$

Then $h(0)=G_{\text {in }}+f_{5}\left(\tau_{0} f_{1}(0)\right)>0$. Also, by the various properties of $f_{2}, f_{4}$ and $f_{5}$ listed in Appendix B, it is clear that $h(G)<0$ for $G$ sufficiently large. It is also straightforward to show that $h^{\prime}(G)<0$ for all $G>0$. Hence there exists precisely one root $G^{*}>0$ of $(2.8)$, and therefore there is one equilibrium $\left(I^{*}, G^{*}\right)$ of $(2.1)$. 


\section{Global convergence to equilibrium}

In this section we shall provide some conditions under which global convergence of solutions to the equilibrium $\left(I^{*}, G^{*}\right)$ is assured. Our first approach is to use a comparison principle. This approach furnishes a set of conditions which involve the parameter $\tau_{0}$, but not the delay $\tau$. Our second approach, by use of Lyapunov functionals, yields another set of sufficient conditions which do involve the delay $\tau$, yielding further insight into the behaviour of the system.

\subsection{Comparison principle approach}

Solving the first equation of (2.1) for $I(t)$, gives

$$
I(t)=e^{\frac{-t}{\tau_{0}}} I(0)+e^{\frac{-t}{\tau_{0}}} \int_{0}^{t} e^{\frac{s}{\tau_{0}}} f_{1}(G(s)) d s .
$$

Since we are interested in the asymptotic behaviour of the solutions, we shall neglect the first term in the above. Substituting the remaining expression into the second equation of (2.1), we can recast the original model into the form of a single equation

$$
\begin{aligned}
\frac{d G}{d t}= & G_{i n}-f_{2}(G)-q G f_{4}\left(\int_{0}^{t} e^{\frac{-(t-s)}{\tau_{0}}} f_{1}(G(s)) d s\right) \\
& +f_{5}\left(\int_{0}^{t-\tau} e^{\frac{-(t-\tau-s)}{\tau_{0}}} f_{1}(G(s)) d s\right), \quad t>0
\end{aligned}
$$

which now requires as initial data:

$$
G(s)=G_{0}(s), \quad s \in[-\tau, 0]
$$

where $G_{0}(s)$ is a prescribed, continuous, non-negative, initial function with $G_{0}(0)>0$. Although we have reduced the original system (2.1) to a single equation, this has been done at the expense of now having to deal with distributed delay terms. We shall now introduce a definition of sub- and supersolutions appropriate to our problem, and then state a comparison principle which shall be used to prove a theorem on global convergence.

Definition A pair of sub- and supersolutions for (3.1,3.2) is a pair of suitably smooth functions $v$ and $w$ such that:

(i) $v \leqslant w$ for all $t$;

(ii) $v$ and $w$ satisfy

$$
\begin{aligned}
& \frac{d v}{d t} \leqslant G_{i n}-f_{2}(v)-q v f_{4}\left(\int_{0}^{t} e^{\frac{-(t-s)}{\tau_{0}}} f_{1}(\phi(s)) d s\right)+f_{5}\left(\int_{0}^{t-\tau} e^{\frac{-(t-\tau-s)}{\tau_{0}}} f_{1}(\phi(s)) d s\right) \\
& \frac{d w}{d t} \geqslant G_{\text {in }}-f_{2}(w)-q w f_{4}\left(\int_{0}^{t} e^{\frac{-(t-s)}{\tau_{0}}} f_{1}(\phi(s)) d s\right)+f_{5}\left(\int_{0}^{t-\tau} e^{\frac{-(t-\tau-s)}{\tau_{0}}} f_{1}(\phi(s)) d s\right)
\end{aligned}
$$

for all functions $\phi$ such that $v(s) \leqslant \phi(s) \leqslant w(s), s \leqslant t$;

(iii) $v(s) \leqslant G_{0}(s) \leqslant w(s)$ for all $s \in[-\tau, 0]$. 
We shall employ the following comparison principle, which is a consequence of Theorem 3.4 in Redlinger [14]:

Lemma 3.1 If there are sub- and supersolutions $v$ and $w$ for (3.1), (3.2), then there exists a unique solution $G(t)$ of (3.1), (3.2) such that $v(t) \leqslant G(t) \leqslant w(t)$ for all $t$.

Trivially, we have that 0 is a subsolution of (3.1), (3.2). Let us seek a supersolution. Define $\hat{G}$ to be the solution of

$$
\frac{d \hat{G}}{d t}=G_{i n}-f_{2}(\hat{G})-q \hat{G} f_{4}(0)+f_{5}(0), \quad t>0 .
$$

Although this is not a delay equation, we do need to define $\hat{G}$ on the interval $[-\tau, 0]$ because of condition (iii) in the above Definition. For $s \in[-\tau, 0)$, we shall take $\hat{G}(s):=$ $\hat{G}(0):=\max \left\{G_{0}(\tilde{s}), \tilde{s} \in[-\tau, 0]\right\}$. Conditions (i) and (iii) of the Definition are then trivially satisfied. Condition (ii) will be satisfied if

$$
q \hat{G} f_{4}(0)-f_{5}(0) \leqslant q \hat{G} \underbrace{f_{4}\left(\int_{0}^{t} e^{\frac{-(t-s)}{\tau_{0}}} f_{1}(\phi(s)) d s\right)}_{\geqslant f_{4}(0)}-\underbrace{f_{5}\left(\int_{0}^{t-\tau} e^{\frac{-(t-\tau-s)}{\tau_{0}}} f_{1}(\phi(s)) d s\right)}_{\leqslant f_{5}(0)}
$$

for all functions $\phi$ with $0 \leqslant \phi(s) \leqslant \hat{G}(s), s \leqslant t$, and (3.3) holds because of the monotonicity properties of $f_{4}$ and $f_{5}$. Therefore, $(0, \hat{G})$ is a sub- supersolution pair and thus there exists a unique solution $G(t)$ to $(3.1,3.2)$ such that $0 \leqslant G(t) \leqslant \hat{G}(t)$ for all $t$.

Our main theorem of this subsection is the following.

Theorem 3.2 Let the $f_{i}$ satisfy the assumptions listed in Appendix B, and suppose that the simultaneous equations

$$
\begin{aligned}
& G_{\text {in }}-f_{2}(x)-q x f_{4}\left(\tau_{0} f_{1}(y)\right)+f_{5}\left(\tau_{0} f_{1}(y)\right)=0 \\
& G_{\text {in }}-f_{2}(y)-q y f_{4}\left(\tau_{0} f_{1}(x)\right)+f_{5}\left(\tau_{0} f_{1}(x)\right)=0
\end{aligned}
$$

have no solution in the first quadrant other than $x=y=G^{*}$. Then the solution $G(t)$ of (3.1), (3.2) satisfies

$$
\lim _{t \rightarrow \infty} G(t)=G^{*}
$$

Remark Later, we shall discuss under what circumstances the hypothesis of this theorem is likely to be satisfied.

\section{Proof Let}

$$
I=\left[\liminf _{t \rightarrow \infty} G(t), \limsup _{t \rightarrow \infty} G(t)\right] .
$$

To prove the theorem, it suffices to show that $I=\left\{G^{*}\right\}$. Now

$$
\limsup _{t \rightarrow \infty} G(t) \leqslant \lim _{t \rightarrow \infty} \hat{G}(t)=: v_{0} .
$$


Therefore, $I \subset\left[0, v_{0}\right]$. Furthermore, $v_{0}$ satisfies

$$
G_{\text {in }}-f_{2}\left(v_{0}\right)-q v_{0} f_{4}(0)+f_{5}(0)=0 .
$$

We now improve the subsolution. Let $\varepsilon>0$. By (3.6), there exists $t_{1}>1$ such that

$$
G(t) \leqslant v_{0}+\varepsilon \text { for all } t \geqslant t_{1}-1
$$

and there exists $t_{2}>t_{1}+\tau$ such that

$$
\int_{t-t_{1}}^{t} e^{-s / \tau_{0}} d s<\varepsilon \text { for all } t \geqslant t_{2}-\tau
$$

Since $G(t)$ is majorized by $\hat{G}(t)$, and the latter is a monotone function (it satisfies a one-dimensional autonomous ODE), we can say that, for all $t \geqslant-\tau$,

$$
G(t) \leqslant \tilde{M}_{G}:=\max \left(v_{0}, \max \left\{G_{0}(\tilde{s}), \tilde{s} \in[-\tau, 0]\right\}\right) .
$$

Introduce the function

$$
z^{(1)}(t)= \begin{cases}\tilde{M}_{G}, & -\tau \leqslant t \leqslant t_{1}-1 \\ v_{0}+\varepsilon+\left(\tilde{M}_{G}-v_{0}-\varepsilon\right)\left(t_{1}-t\right), & t_{1}-1<t<t_{1} \\ v_{0}+\varepsilon, & t \geqslant t_{1}\end{cases}
$$

and also the 'cut-off' operator

$$
\left(A^{(1)} G\right)(t)=\max \left(0, \min \left(G(t), z^{(1)}(t)\right)\right) .
$$

We see that $G(t) \leqslant z^{(1)}(t)$ for all $t$ since $0 \leqslant G(t) \leqslant v_{0}+\varepsilon$ for $t \geqslant t_{1}-1$ and $0 \leqslant G(t) \leqslant \tilde{M}_{G}$ for all $t$ (in particular for $t<t_{1}-1$ ). Hence, $A^{(1)} G=G$ and therefore replacing $G$ by $A^{(1)} G$ in the delay terms of equation (3.1) leaves the solution unaltered. Of course, we shall also carry out this replacement in the definition of sub- and supersolutions, with the effect that the functions $\phi$ in that definition are 'cut off' by the operator $A^{(1)}$. This leads to an improved subsolution.

It is straightforward to see that the solution of (3.1), (3.2) satisfies $G(t)>0$ for all $t>0$. Therefore, if

$$
\delta_{1}=\frac{1}{2} \min \left\{G(t): \frac{1}{2} t_{2} \leqslant t \leqslant t_{2}\right\}
$$

then $\delta_{1}>0$. Define the function $v_{1}$ by

$$
\begin{gathered}
v_{1}(t)= \begin{cases}0, & -\tau \leqslant t \leqslant \frac{1}{2} t_{2} \\
\frac{\delta_{1}}{t_{2}}\left(2 t-t_{2}\right), & \frac{1}{2} t_{2}<t \leqslant t_{2}\end{cases} \\
\dot{v}_{1}=G_{i n}-f_{2}\left(v_{1}\right)-q v_{1} f_{4}\left(\varepsilon f_{1}\left(\tilde{M}_{G}\right)+\tau_{0} f_{1}\left(v_{0}+\varepsilon\right)\right) \\
+f_{5}\left(\varepsilon f_{1}\left(\tilde{M}_{G}\right)+\tau_{0} f_{1}\left(v_{0}+\varepsilon\right)\right),
\end{gathered}
$$

We claim that $v_{1}$ and $w_{1} \equiv \tilde{M}_{G}$ are sub- and supersolutions for (3.1), (3.2). On [0,t $t_{2}$ we have $v_{1}(t)<G(t)$, so the first inequality of (ii) in the definition of a subsolution need only 
hold for $t>t_{2}$. Therefore, we need to show that $v_{1}<\tilde{M}_{G}$ for all $t \geqslant 0$ and that, for $t>t_{2}$,

$$
\begin{aligned}
& q v_{1} f_{4}\left(\int_{0}^{t} e^{\frac{-(t-s)}{\tau_{0}}} f_{1}\left(\left(A^{(1)} \phi\right)(s)\right) d s\right)-f_{5}\left(\int_{0}^{t-\tau} e^{\frac{-(t-\tau-s)}{\tau_{0}}} f_{1}\left(\left(A^{(1)} \phi\right)(s)\right) d s\right) \\
& \leqslant q v_{1} f_{4}\left(\varepsilon f_{1}\left(\tilde{M}_{G}\right)+\tau_{0} f_{1}\left(v_{0}+\varepsilon\right)\right)-f_{5}\left(\varepsilon f_{1}\left(\tilde{M}_{G}\right)+\tau_{0} f_{1}\left(v_{0}+\epsilon\right)\right)
\end{aligned}
$$

for all functions $\phi$ with $v_{1} \leqslant \phi \leqslant \tilde{M}_{G}$. Note that $A^{(1)} \phi \leqslant z^{(1)}$ and consequently

$$
\begin{aligned}
& s \in\left[0, t_{1}\right] \Rightarrow\left(A^{(1)} \phi\right)(s) \leqslant \tilde{M}_{G}, \\
& s \in\left[t_{1}, t\right] \Rightarrow\left(A^{(1)} \phi\right)(s) \leqslant v_{0}+\varepsilon .
\end{aligned}
$$

Thus, for $t>t_{2}$,

$$
\begin{aligned}
& q v_{1} f_{4}\left(\int_{0}^{t} e^{\frac{-(t-s)}{\tau_{0}}} f_{1}\left(\left(A^{(1)} \phi\right)(s)\right) d s\right) \\
& =q v_{1} f_{4}\left(\int_{0}^{t_{1}} e^{\frac{-(t-s)}{\tau_{0}}} f_{1}\left(\left(A^{(1)} \phi\right)(s)\right) d s+\int_{t_{1}}^{t} e^{\frac{-(t-s)}{\tau_{0}}} f_{1}\left(\left(A^{(1)} \phi\right)(s)\right) d s\right) \\
& \quad \leqslant q v_{1} f_{4}\left(f_{1}\left(\tilde{M}_{G}\right) \int_{0}^{t_{1}} e^{\frac{-(t-s)}{\tau_{0}}} d s+f_{1}\left(v_{0}+\varepsilon\right) \int_{t_{1}}^{t} e^{\frac{-(t-s)}{\tau_{0}}} d s\right) \\
& \quad=q v_{1} f_{4}\left(f_{1}\left(\tilde{M}_{G}\right) \int_{t-t_{1}}^{t} e^{\frac{-s}{\tau_{0}}} d s+f_{1}\left(v_{0}+\varepsilon\right) \int_{0}^{t-t_{1}} e^{\frac{-s}{\tau_{0}}} d s\right) \\
& \quad<q v_{1} f_{4}\left(\varepsilon f_{1}\left(\tilde{M}_{G}\right)+\tau_{0} f_{1}\left(v_{0}+\varepsilon\right)\right)
\end{aligned}
$$

and, similarly,

$$
f_{5}\left(\int_{0}^{t-\tau} e^{\frac{-(t-\tau-s)}{\tau_{0}}} f_{1}\left(\left(A^{(1)} \phi\right)(s)\right) d s\right)>f_{5}\left(\varepsilon f_{1}\left(\tilde{M}_{G}\right)+\tau_{0} f_{1}\left(v_{0}+\varepsilon\right)\right) .
$$

Hence inequality (3.8) is satisfied.

As $t \rightarrow \infty, v_{1}$ tends to a limit $\mu=\mu_{1}(\varepsilon)$ satisfying the equation

$$
\begin{aligned}
p(\mu ; \varepsilon)= & G_{i n}-f_{2}(\mu)-q \mu f_{4}\left(\varepsilon f_{1}\left(\tilde{M}_{G}\right)+\tau_{0} f_{1}\left(v_{0}+\varepsilon\right)\right) \\
& +f_{5}\left(\varepsilon f_{1}\left(\tilde{M}_{G}\right)+\tau_{0} f_{1}\left(v_{0}+\varepsilon\right)\right)=0 .
\end{aligned}
$$

Now $p^{\prime}(\mu ; \varepsilon)<0$ for all $\mu \geqslant 0$. Also, $p(0 ; \varepsilon)>0$ and $p(\mu ; \varepsilon)<0$ for sufficiently large $\mu$. Therefore, $p(\mu ; \varepsilon)=0$ has one strictly positive root $\mu=\mu_{1}(\varepsilon)$ which is a continuous function of $\varepsilon$, and $p(\mu ; \varepsilon)>0$ when $\mu \in\left(0, \mu_{1}(\varepsilon)\right)$ and $p(\mu ; \varepsilon)<0$ when $\mu>\mu_{1}(\varepsilon)$. It follows that

$$
\lim _{t \rightarrow \infty} v_{1}(t ; \varepsilon)=\mu_{1}(\varepsilon) \text {. }
$$

We still need to check that $v_{1}<\tilde{M}_{G}$ for all $t \geqslant 0$. Now, using (2.8),

$$
\begin{aligned}
p\left(G^{*} ; \varepsilon\right)= & q G^{*} f_{4}\left(\tau_{0} f_{1}\left(G^{*}\right)\right)-q G^{*} f_{4}\left(\varepsilon f_{1}\left(\tilde{M}_{G}\right)+\tau_{0} f_{1}\left(v_{0}+\varepsilon\right)\right) \\
& +f_{5}\left(\varepsilon f_{1}\left(\tilde{M}_{G}\right)+\tau_{0} f_{1}\left(v_{0}+\varepsilon\right)\right)-f_{5}\left(\tau_{0} f_{1}\left(G^{*}\right)\right) .
\end{aligned}
$$


If we can show $G^{*} \leqslant v_{0}$ then $p\left(G^{*} ; \varepsilon\right)<0$. Assume, for contradiction, that $G^{*}>v_{0}$. Then from (3.7) and (2.8) it is necessary that

$$
\begin{aligned}
0= & \underbrace{f_{2}\left(G^{*}\right)-f_{2}\left(v_{0}\right)}_{>0}+\underbrace{q G^{*} f_{4}\left(\tau_{0} f_{1}\left(G^{*}\right)\right)-q v_{0} f_{4}(0)}_{>0} \\
& +\underbrace{f_{5}(0)-f_{5}\left(\tau_{0} f_{1}\left(G^{*}\right)\right)}_{>0},
\end{aligned}
$$

a contradiction. Hence $G^{*} \leqslant v_{0}$ and $p\left(G^{*} ; \varepsilon\right)<0$. Therefore $\mu_{1}(\varepsilon)<G^{*} \leqslant v_{0} \leqslant \tilde{M}_{G}$. Since $v_{1}$ approaches its limit $\mu_{1}(\varepsilon)$ monotonically, our observations are sufficient to ensure that $v_{1} \leqslant \tilde{M}_{G}$ for all $t \geqslant 0$. So $v_{1}$ is a subsolution and, from Lemma 3.1,

$$
v_{1}(t ; \varepsilon) \leqslant G(t) \leqslant \tilde{M}_{G} .
$$

Letting $\varepsilon \rightarrow 0$ and writing $\mu_{1}(0)=\mu_{1}$ we conclude that $I \subset\left[\mu_{1}, v_{0}\right]$, where $\mu_{1}$ satisfies

$$
G_{\text {in }}-f_{2}\left(\mu_{1}\right)-q \mu_{1} f_{4}\left(\tau_{0} f_{1}\left(v_{0}\right)\right)+f_{5}\left(\tau_{0} f_{1}\left(v_{0}\right)\right)=0 .
$$

One can then improve this to $I \subset\left[\mu_{1}, v_{1}\right]$ where $v_{1}$ is defined in terms of $\mu_{0}$. Carrying on with this process (the details are similar to those already presented), one finds that $I \subset\left[\mu_{n}, v_{n}\right]$ for each $n \in \mathbb{N}$, where $\left(\mu_{n}\right)$ and $\left(v_{n}\right)$ are defined by

$$
\begin{aligned}
& G_{i n}-f_{2}\left(\mu_{n+1}\right)-q \mu_{n+1} f_{4}\left(\tau_{0} f_{1}\left(v_{n}\right)\right)+f_{5}\left(\tau_{0} f_{1}\left(v_{n}\right)\right)=0, \\
& G_{i n}-f_{2}\left(v_{n+1}\right)-q v_{n+1} f_{4}\left(\tau_{0} f_{1}\left(\mu_{n}\right)\right)+f_{5}\left(\tau_{0} f_{1}\left(\mu_{n}\right)\right)=0 .
\end{aligned}
$$

We shall show by induction that

$$
0<\mu_{0} \leqslant \mu_{1} \leqslant \cdots \leqslant \mu_{n}<G^{*}<v_{n} \leqslant v_{n-1} \leqslant \cdots \leqslant v_{1} \leqslant v_{0} .
$$

Assuming (3.11) is true (inductive hypothesis), then we need to show

$$
\mu_{n} \leqslant \mu_{n+1}<G^{*}
$$

and

$$
G^{*}<v_{n+1} \leqslant v_{n}
$$

We shall show only the former. Now, $\mu_{n+1}$ is the root $x$ of

$$
F(x)=G_{\text {in }}-f_{2}(x)-q x f_{4}\left(\tau_{0} f_{1}\left(v_{n}\right)\right)+f_{5}\left(\tau_{0} f_{1}\left(v_{n}\right)\right)=0
$$

and therefore (3.12) is satisfied if $F\left(\mu_{n}\right) \geqslant 0$ and $F\left(G^{*}\right)<0$. Now

$$
\begin{aligned}
F\left(\mu_{n}\right)= & G_{i n}-f_{2}\left(\mu_{n}\right)-q \mu_{n} f_{4}\left(\tau_{0} f_{1}\left(v_{n}\right)\right)+f_{5}\left(\tau_{0} f_{1}\left(v_{n}\right)\right) \\
= & q \mu_{n} f_{4}\left(\tau_{0} f_{1}\left(v_{n-1}\right)\right)-f_{5}\left(\tau_{0} f_{1}\left(v_{n-1}\right)+f_{5}\left(\tau_{0} f_{1}\left(v_{n}\right)\right)\right. \\
& -q \mu_{n} f_{4}\left(\tau_{0} f_{1}\left(v_{n}\right)\right) \\
= & q \mu_{n} \underbrace{\left(f_{4}\left(\tau_{0} f_{1}\left(v_{n-1}\right)\right)-f_{4}\left(\tau_{0} f_{1}\left(v_{n}\right)\right)\right)}_{\geqslant 0} \\
& +\underbrace{f_{5}\left(\tau_{0} f_{1}\left(v_{n}\right)\right)-f_{5}\left(\tau_{0} f_{1}\left(v_{n-1}\right)\right)}_{\geqslant 0} \\
\geqslant & 0 \quad \begin{aligned}
\text { since } v_{n} \leqslant v_{n-1} .
\end{aligned}
\end{aligned}
$$


The proof that $F\left(G^{*}\right)<0$ is similar. Hence (3.12) is satisfied. Similarly, we can show that (3.13) holds, proving (3.11). We can deduce that there exist the limits

$$
\mu=\lim _{n \rightarrow \infty} \mu_{n} \quad \text { and } \quad v=\lim _{n \rightarrow \infty} v_{n}
$$

and, from (3.10) with $n \rightarrow \infty$,

$$
\begin{aligned}
& G_{i n}-f_{2}(\mu)-q \mu f_{4}\left(\tau_{0} f_{1}(v)\right)+f_{5}\left(\tau_{0} f_{1}(v)\right)=0, \\
& G_{i n}-f_{2}(v)-q v f_{4}\left(\tau_{0} f_{1}(\mu)\right)+f_{5}\left(\tau_{0} f_{1}(\mu)\right)=0 .
\end{aligned}
$$

By the hypothesis of the theorem, these equations have only the solution $\mu=v=G^{*}$. Since $I \subset[\mu, v]$, it follows that $I=\left\{G^{*}\right\}$ and the proof of the theorem is complete.

As promised earlier, we shall now discuss the circumstances under which the simultaneous equations $(3.4,3.5)$ are likely to have only the solution $x=y=G^{*}$. With only the general assumptions on the $f_{i}$ listed in Appendix B to work with, it is difficult to ascertain precisely the circumstances, but by some simple graphical arguments we can make some very useful comments.

Equation (3.4) defines a curve $y=y(x)$ in the $(x, y)$ plane. Only the first quadrant is of interest. From the properties of the $f_{i}$ it is easy to see that this curve intersects the $x$-axis precisely once, but does not intersect the $y$-axis. Furthermore, by implicitly differentiating (3.4) with respect to $x$, with $y=y(x)$, we find that

$$
y^{\prime}(x)=\frac{f_{2}^{\prime}(x)+q f_{4}\left(\tau_{0} f_{1}(y)\right)}{\tau_{0} f_{1}^{\prime}(y)\left\{f_{5}^{\prime}\left(\tau_{0} f_{1}(y)\right)-q x f_{4}^{\prime}\left(\tau_{0} f_{1}(y)\right)\right\}}
$$

so that, since $f_{5}$ is decreasing, $y(x)$ is always decreasing along the curve. The second equation (3.5) defines a curve that is the mirror image, in the line $y=x$, of the curve we have just been discussing.

The graphs shown in Fig. 1 illustrate two possibilities. In one of these the two curves have only the $x=y=G^{*}$ intersection while, in the other, there are two additional intersections so that the hypothesis of Theorem 3.2 is not satisfied. On a first glance, what appears to distinguish the two cases is the slopes at the intersection with $y=x$. It is actually not as simple as this; one can imagine that curve 1 could be very steep until just after its intersection with $y=x$, and then suddenly swing round and intersect curve 2 in two further places below $y=x$. However, MAPLE plots of the two curves for the case when the $f_{i}$ are given by expressions (4.2), (4.3), (4.4) and (4.6) of Appendix A suggest that this never happens and that for all biologically reasonable sets of parameter values it is indeed the slopes at the intersection with $y=x$ that distinguishes the two cases. Examining the slopes at $x=y=G^{*}$, we require the slope of curve 1 at that point to be less than -1 . Equation (3.14) then gives us

$$
f_{2}^{\prime}\left(G^{*}\right)+q f_{4}\left(\tau_{0} f_{1}\left(G^{*}\right)\right)+\tau_{0} f_{1}^{\prime}\left(G^{*}\right)\left\{f_{5}^{\prime}\left(\tau_{0} f_{1}\left(G^{*}\right)\right)-q G^{*} f_{4}^{\prime}\left(\tau_{0} f_{1}\left(G^{*}\right)\right)\right\}>0 .
$$

These observations suggest that if (3.15) holds then the hypothesis of Theorem 3.2 is satisfied for realistic $f_{i}$ and for realistic parameter values. 

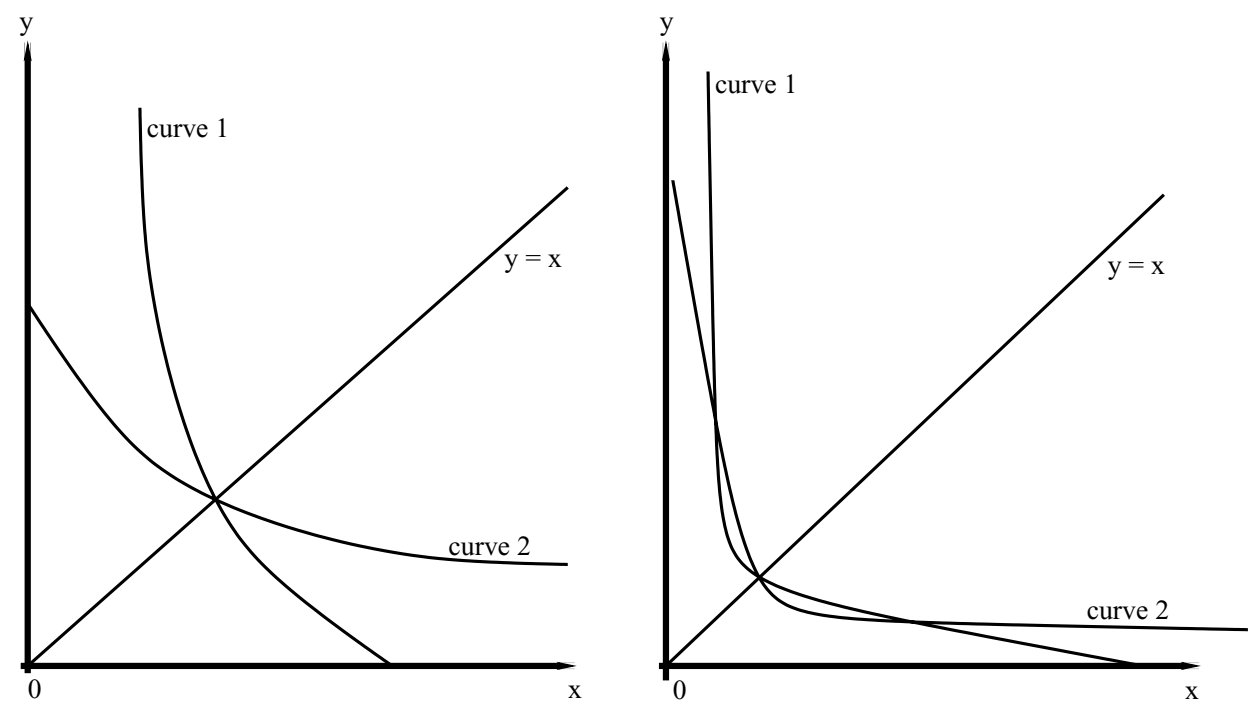

FIGURE 1. Qualitative sketches of the curves defined by equations (3.4) and (3.5) showing two possibilities, only one of which satisfies the hypothesis of Theorem 3.2.

\subsection{Lyapunov functional approach}

In the approach to be described in this section, we shall work with the original problem (2.1). The functions $f_{i}$ shall take the expressions given in Appendix A, and our aim is to study how the global stability of the equilibrium $\left(G^{*}, I^{*}\right)$ of $(2.1)$ depends on $\tau_{0}$ and $\tau$. It is inconvenient and unnecessary to carry the exact expressions for the $f_{i}$ through all the analysis; we shall call upon the actual expressions only as necessary.

Applying the transformation

$$
G=G^{*}+u, \quad I=I^{*}+v
$$

to system (2.1) gives

$$
\begin{aligned}
& \dot{u}=-u f_{2}^{\prime}\left(G^{*}+\theta_{2} u\right)-u q f_{4}\left(I^{*}\right)-v f_{4}^{\prime}\left(I^{*}+\theta_{4} v\right) q\left(G^{*}+u\right)+v(t-\tau) f_{5}^{\prime}\left(I^{*}+\theta_{5} v(t-\tau)\right) \\
& \dot{v}=u f_{1}^{\prime}\left(G^{*}+\theta_{1} u\right)-\frac{1}{\tau_{0}} v
\end{aligned}
$$

where the $\theta_{i}$ come from applications of Taylor's theorem with remainder, for example,

$$
f_{2}\left(G^{*}+u(t)\right)=f_{2}\left(G^{*}\right)+u(t) f_{2}^{\prime}\left(G^{*}+u(t) \theta_{1}(u(t))\right)
$$

where, for all $t, \theta_{1}$ is between 0 and 1 . We do not need to keep track of the dependence of the $\theta_{i}$ on the state variables or the times at which these are evaluated (for example, $\theta_{1}(u(t))$ and $\theta_{1}(u(s))$ shall both appear simply as $\theta_{1}$ in our analysis). All that we need to know about the $\theta_{i}$ is that they are always between 0 and 1 .

In the new system (3.16) the equilibrium of interest is $u=v=0$. In what follows, $u$ and $v$ are always evaluated at time $t$ except where otherwise shown. In the following analysis, 
we shall several times make use of the inequality

$$
x y \leqslant \frac{1}{2} \varepsilon_{i} x^{2}+\frac{1}{2 \varepsilon_{i}} y^{2}
$$

with suitably chosen $\varepsilon_{i}$. We shall need an upper bound on $G(t)$. Since we are working with the system (2.1), the upper bound given by

$$
G(t) \leqslant M_{G}:=\max \left\{G(0), \frac{1}{q f_{4}(0)}\left(G_{i n}+f_{5}(0)\right\}\right.
$$

is valid here. Define

$$
U_{1}(u, v)=\frac{1}{2} u^{2}+\frac{1}{2} \omega v^{2}>0
$$

where $\omega>0$ is to be chosen later. Along the solutions of (3.16),

$$
\begin{aligned}
& \dot{U}_{1}=-\left[f_{2}^{\prime}\left(G^{*}+\theta_{2} u\right)+q f_{4}\left(I^{*}\right)\right] u^{2}-\frac{\omega}{\tau_{0}} v^{2}-u v f_{4}^{\prime}\left(I^{*}+\theta_{4} v\right) q\left(G^{*}+u\right) \\
& +\omega u v f_{1}^{\prime}\left(G^{*}+\theta_{1} u\right)+u v f_{5}^{\prime}\left(I^{*}+\theta_{5} v(t-\tau)\right) \\
& +u f_{5}^{\prime}\left(I^{*}+\theta_{5} v(t-\tau)\right)[v(t-\tau)-v(t)] \\
& =-\left[f_{2}^{\prime}\left(G^{*}+\theta_{2} u\right)+q f_{4}\left(I^{*}\right)\right] u^{2}-\frac{\omega}{\tau_{0}} v^{2}-u v f_{4}^{\prime}\left(I^{*}+\theta_{4} v\right) q\left(G^{*}+u\right) \\
& +\omega u v f_{1}^{\prime}\left(G^{*}+\theta_{1} u\right)+u v f_{5}^{\prime}\left(I^{*}+\theta_{5} v(t-\tau)\right) \\
& -u f_{5}^{\prime}\left(I^{*}+\theta_{5} v(t-\tau)\right) \int_{t-\tau}^{t} \dot{v}(s) d s \\
& =-\left[f_{2}^{\prime}\left(G^{*}+\theta_{2} u\right)+q f_{4}\left(I^{*}\right)\right] u^{2}-\frac{\omega}{\tau_{0}} v^{2}-u v f_{4}^{\prime}\left(I^{*}+\theta_{4} v\right) q\left(G^{*}+u\right) \\
& +\omega u v f_{1}^{\prime}\left(G^{*}+\theta_{1} u\right)+u v f_{5}^{\prime}\left(I^{*}+\theta_{5} v(t-\tau)\right) \\
& -u f_{5}^{\prime}\left(I^{*}+\theta_{5} v(t-\tau)\right) \int_{t-\tau}^{t}\left\{u(s) f_{1}^{\prime}\left(G^{*}+\theta_{1} u(s)\right)-\frac{1}{\tau_{0}} v(s)\right\} d s \\
& \leqslant-\left[f_{2}^{\prime}\left(G^{*}+\theta_{2} u\right)+q f_{4}\left(I^{*}\right)\right] u^{2}-\frac{\omega}{\tau_{0}} v^{2} \\
& +\frac{1}{2}\left(\varepsilon_{1} u^{2}+\frac{v^{2}}{\varepsilon_{1}}\right) f_{4}^{\prime}\left(I^{*}+\theta_{4} v\right) q\left(G^{*}+u\right)+\frac{\omega}{2}\left(\varepsilon_{2} u^{2}+\frac{v^{2}}{\varepsilon_{2}}\right) f_{1}^{\prime}\left(G^{*}+\theta_{1} u\right) \\
& +\frac{1}{2}\left(\varepsilon_{3} u^{2}+\frac{v^{2}}{\varepsilon_{3}}\right)\left|f_{5}^{\prime}\left(I^{*}+\theta_{5} v(t-\tau)\right)\right| \\
& +\left|f_{5}^{\prime}\left(I^{*}+\theta_{5} v(t-\tau)\right)\right| \int_{t-\tau}^{t}\left\{f_{1}^{\prime}\left(G^{*}+\theta_{1} u(s)\right)|u(s)||u(t)|+\frac{1}{\tau_{0}}|v(s)||u(t)|\right\} d s \\
& \leqslant-\left[f_{2}^{\prime}\left(G^{*}+\theta_{2} u\right)+q f_{4}\left(I^{*}\right)\right] u^{2}-\frac{\omega}{\tau_{0}} v^{2}+\frac{1}{2}\left(\varepsilon_{1} u^{2}+\frac{v^{2}}{\varepsilon_{1}}\right) f_{4}^{\prime}\left(I^{*}+\theta_{4} v\right) q\left(G^{*}+u\right) \\
& +\frac{\omega}{2}\left(\varepsilon_{2} u^{2}+\frac{v^{2}}{\varepsilon_{2}}\right) f_{1}^{\prime}\left(G^{*}+\theta_{1} u\right)+\frac{1}{2}\left(\varepsilon_{3} u^{2}+\frac{v^{2}}{\varepsilon_{3}}\right)\left|f_{5}^{\prime}\left(I^{*}+\theta_{5} v(t-\tau)\right)\right| \\
& +\frac{1}{2}\left|f_{5}^{\prime}\left(I^{*}+\theta_{5} v(t-\tau)\right)\right| f_{1}^{\prime}\left(C_{1} V_{g}\right) \int_{t-\tau}^{t}\left\{\frac{1}{\varepsilon_{4}} u^{2}(s)+\varepsilon_{4} u^{2}(t)\right\} d s \\
& +\frac{1}{2 \tau_{0}}\left|f_{5}^{\prime}\left(I^{*}+\theta_{5} v(t-\tau)\right)\right| \int_{t-\tau}^{t}\left\{\frac{v^{2}(s)}{\varepsilon_{5}}+\varepsilon_{5} u^{2}(t)\right\} d s
\end{aligned}
$$




$$
\begin{aligned}
\leqslant & -\left[f_{2}^{\prime}\left(G^{*}+\theta_{2} u\right)+q f_{4}\left(I^{*}\right)-\frac{1}{2} \omega \varepsilon_{2} f_{1}^{\prime}\left(G^{*}+\theta_{1} u\right)\right. \\
& -\frac{1}{2} \varepsilon_{1} f_{4}^{\prime}\left(I^{*}+\theta_{4} v\right) q\left(G^{*}+u\right)-\frac{1}{2} \varepsilon_{3}\left|f_{5}^{\prime}\left(C_{5} V_{i}\right)\right| \\
& \left.-\frac{1}{2} \varepsilon_{4}\left|f_{5}^{\prime}\left(C_{5} V_{i}\right)\right| f_{1}^{\prime}\left(C_{1} V_{g}\right) \tau-\frac{1}{2 \tau_{0}} \varepsilon_{5}\left|f_{5}^{\prime}\left(C_{5} V_{i}\right)\right| \tau\right] u^{2} \\
& -\left[\frac{\omega}{\tau_{0}}-\frac{1}{2 \varepsilon_{1}} f_{4}^{\prime}\left(I^{*}+\theta_{4} v\right) q\left(G^{*}+u\right)-\frac{\omega}{2 \varepsilon_{2}} f_{1}^{\prime}\left(G^{*}+\theta_{1} u\right)-\frac{1}{2 \varepsilon_{3}}\left|f_{5}^{\prime}\left(C_{5} V_{i}\right)\right|\right] v^{2} \\
& +\frac{1}{2 \varepsilon_{4}}\left|f_{5}^{\prime}\left(C_{5} V_{i}\right)\right| f_{1}^{\prime}\left(C_{1} V_{g}\right) \int_{t-\tau}^{t} u^{2}(s) d s+\frac{1}{2 \tau_{0} \varepsilon_{5}}\left|f_{5}^{\prime}\left(C_{5} V_{i}\right)\right| \int_{t-\tau}^{t} v^{2}(s) d s .
\end{aligned}
$$

In the above estimates we have used the fact that $f_{1}^{\prime}(G)$ is maximised at $G=C_{1} V_{g}$ and that $\left|f_{5}^{\prime}(I)\right|$ is maximised at $I=C_{5} V_{i}$. Similarly, in the following analysis, we shall use that $f_{4}^{\prime}(I)$ is maximised at $I=A$, where $A$ is the quantity defined in the statement of Theorem 3.3 below, and that $f_{2}^{\prime}(G) \geqslant f_{2}^{\prime}\left(M_{G}\right)>0$, since $G(t)$ is bounded by $M_{G}$. Now define

$$
W_{1}=\frac{1}{2 \varepsilon_{4}}\left|f_{5}^{\prime}\left(C_{5} V_{i}\right)\right| f_{1}^{\prime}\left(C_{1} V_{g}\right) \int_{t-\tau}^{t} \int_{z}^{t} u^{2}(s) d s d z
$$

and

$$
W_{2}=\frac{1}{2 \tau_{0} \varepsilon_{5}}\left|f_{5}^{\prime}\left(C_{5} V_{i}\right)\right| \int_{t-\tau}^{t} \int_{z}^{t} v^{2}(s) d s d z .
$$

If $V=U_{1}+W_{1}+W_{2}$, then

$$
\begin{aligned}
\dot{V} \leqslant & -\left[f_{2}^{\prime}\left(M_{G}\right)+q f_{4}\left(I^{*}\right)\right. \\
& -\frac{1}{2} \varepsilon_{1} f_{4}^{\prime}(A) q M_{G}-\frac{1}{2} \omega \varepsilon_{2} f_{1}^{\prime}\left(C_{1} V_{g}\right) \\
& \left.-\frac{1}{2}\left|f_{5}^{\prime}\left(C_{5} V_{i}\right)\right|\left(\varepsilon_{3}+\tau\left(\varepsilon_{4} f_{1}^{\prime}\left(C_{1} V_{g}\right)+\frac{1}{\varepsilon_{4}} f_{1}^{\prime}\left(C_{1} V_{g}\right)+\frac{\varepsilon_{5}}{\tau_{0}}\right)\right)\right] u^{2} \\
& -\left[\frac{\omega}{\tau_{0}}-\frac{1}{2 \varepsilon_{1}} f_{4}^{\prime}(A) q M_{G}-\frac{\omega}{2 \varepsilon_{2}} f_{1}^{\prime}\left(C_{1} V_{g}\right)\right. \\
& \left.-\frac{1}{2}\left|f_{5}^{\prime}\left(C_{5} V_{i}\right)\right|\left(\frac{1}{\varepsilon_{3}}+\frac{\tau}{\varepsilon_{5} \tau_{0}}\right)\right] v^{2} .
\end{aligned}
$$

For $V$ to be a Lyapunov functional we require $\dot{V}<0$ when $(u, v) \neq(0,0)$. This is satisfied provided that the square bracketed coefficients of $u^{2}$ and $v^{2}$ in the above expression are both strictly positive. To maximise the range of $\tau$ for which stability is assured, it is clear that we need to minimise $\varepsilon_{4}+1 / \varepsilon_{4}$, and thus we choose $\varepsilon_{4}=1$. We shall also choose

$$
\varepsilon_{5}=\left|f_{5}^{\prime}\left(C_{5} V_{i}\right)\right| \tau / \omega
$$

We then seek to choose the remaining $\varepsilon_{i}$ and $\omega$ so as to have

$$
\begin{aligned}
& f_{2}^{\prime}\left(M_{G}\right)+q f_{4}\left(I^{*}\right)-\frac{1}{2} \varepsilon_{1} f_{4}^{\prime}(A) q M_{G}-\frac{1}{2} \omega \varepsilon_{2} f_{1}^{\prime}\left(C_{1} V_{g}\right) \\
& \quad-\frac{1}{2}\left|f_{5}^{\prime}\left(C_{5} V_{i}\right)\right|\left(\varepsilon_{3}+2 \tau f_{1}^{\prime}\left(C_{1} V_{g}\right)+\frac{\tau^{2}}{\omega \tau_{0}}\left|f_{5}^{\prime}\left(C_{5} V_{i}\right)\right|\right)>0
\end{aligned}
$$


and

$$
\frac{\omega}{2 \tau_{0}}-\frac{1}{2 \varepsilon_{1}} f_{4}^{\prime}(A) q M_{G}-\frac{\omega}{2 \varepsilon_{2}} f_{1}^{\prime}\left(C_{1} V_{g}\right)-\frac{1}{2 \varepsilon_{3}}\left|f_{5}^{\prime}\left(C_{5} V_{i}\right)\right|>0 .
$$

There are various possible choices for the remaining $\varepsilon_{i}$ and $\omega$ (and even for the expression for $\varepsilon_{5}$ above), but most lead to stability conditions that are exceptionally clumsy to state and add little to our understanding. The following theorem arises from particular choices that seem to capture the essence of things.

Theorem 3.3 Let $f_{1}(G)$ be given by (4.2), $f_{2}(G)$ by (4.4), $f_{4}(I)$ by (4.6) and $f_{5}(I)$ by (4.3). Also, let

$$
A=\frac{\left(\frac{\beta+1}{\beta-1}\right)^{-\frac{1}{\beta}} C_{4} V_{i} E t_{i}}{E t_{i}+V_{i}}
$$

and

$$
M_{G}=\max \left\{G(0), \frac{1}{q f_{4}(0)}\left(G_{i n}+f_{5}(0)\right)\right\}
$$

Then the positive equilibrium $\left(G^{*}, I^{*}\right)$ of system (2.1) is globally asymptotically stable for $\tau_{0}$ and $\tau$ sufficiently small that

$$
\begin{aligned}
& f_{2}^{\prime}\left(M_{G}\right)+q f_{4}\left(I^{*}\right)-2 \tau_{0} f_{1}^{\prime}\left(C_{1} V_{g}\right)\left(f_{4}^{\prime}(A) q M_{G}+\left|f_{5}^{\prime}\left(C_{5} V_{i}\right)\right|\right) \\
& \quad-\frac{1}{2}\left|f_{5}^{\prime}\left(C_{5} V_{i}\right)\right|\left(2 \tau f_{1}^{\prime}\left(C_{1} V_{g}\right)+\frac{f_{1}^{\prime}\left(C_{1} V_{g}\right)\left|f_{5}^{\prime}\left(C_{5} V_{i}\right)\right| \tau^{2}}{\left(f_{4}^{\prime}(A) q M_{G}+\left|f_{5}^{\prime}\left(C_{5} V_{i}\right)\right|\right) \tau_{0}}\right)>0
\end{aligned}
$$

$\left(\right.$ recall $\left.I^{*}=\tau_{0} f_{1}\left(G^{*}\right)\right)$

Proof We need to choose $\varepsilon_{1}, \varepsilon_{2}, \varepsilon_{3}$ and $\omega$ so that (3.17) and (3.18) both hold. Let us choose

$$
\varepsilon_{1}=\varepsilon_{2}=\varepsilon_{3}=2 \tau_{0} f_{1}^{\prime}\left(C_{1} V_{g}\right)
$$

Also, let

$$
\omega=\frac{f_{4}^{\prime}(A) q M_{G}+\left|f_{5}^{\prime}\left(C_{5} V_{i}\right)\right|+\xi}{f_{1}^{\prime}\left(C_{1} V_{g}\right)}
$$

with $\xi$ to be chosen. Inequality (3.18) is then satisfied for any $\xi>0$, while inequality (3.17) now reads

$$
\begin{aligned}
& f_{2}^{\prime}\left(M_{G}\right)+q f_{4}\left(I^{*}\right)-2 \tau_{0} f_{1}^{\prime}\left(C_{1} V_{g}\right)\left(f_{4}^{\prime}(A) q M_{G}+\left|f_{5}^{\prime}\left(C_{5} V_{i}\right)\right|+\frac{1}{2} \xi\right) \\
& \quad-\frac{1}{2}\left|f_{5}^{\prime}\left(C_{5} V_{i}\right)\right|\left(2 \tau f_{1}^{\prime}\left(C_{1} V_{g}\right)+\frac{f_{1}^{\prime}\left(C_{1} V_{g}\right)\left|f_{5}^{\prime}\left(C_{5} V_{i}\right)\right| \tau^{2}}{\left(f_{4}^{\prime}(A) q M_{G}+\left|f_{5}^{\prime}\left(C_{5} V_{i}\right)\right|+\xi\right) \tau_{0}}\right)>0 .
\end{aligned}
$$

Since (3.19) holds, we can obviously choose $\xi>0$ so that (3.20) holds. The proof is complete.

\section{Conclusion}

The two approaches employed in this paper for establishing sufficient conditions for global convergence to equilibrium have yielded two sets of sufficient conditions which involve 
different parameters of the problem. The conditions generated by the comparison principle approach are somewhat implicit, requiring a certain pair of simultaneous equations to have only a certain known solution. Graphical considerations and MAPLE experiments describe the circumstances in which these conditions are likely to hold, suggesting in particular that they hold if the parameter $\tau_{0}$, which measures the timescale on which insulin degrades, is small. The conditions provided by the comparison principle approach do not involve the parameter $\tau$, which measures the time delay between the appearance of insulin in the plasma and its resultant suppressive effect on the rate of glucose production. If the conditions generated by the comparison principle approach hold, then global stability is assured independently of the value of $\tau$. In situations when a delay is incapable of destabilising an equilibrium however large it is, the delay is sometimes said to be harmless.

The Lyapunov functional approach leads to a sufficient condition for global stability that involves the parameter $\tau$, and therefore the role of $\tau$ is discovered to some extent. Again, the conditions are sufficient but not necessary. Note that in the Lyapunov functional approach we have used the expressions for the functions $f_{i}$ that previous investigators have used (see Sturis et al. [19]). However, in fact only certain particular properties of these functions are used, most notably, the maximum values of their derivatives.

The sufficient conditions for global stability produced by the two approaches cease to hold in precisely the circumstances in which other investigators have noted that oscillations appear. It is known (see, for example, Keener \& Sneyd [7]) that a sufficiently large infusion of glucose $\left(G_{i n}\right.$ large) can cause oscillations. Raising $G_{i n}$ has the effect of raising $G^{*}$, as can be seen by examining the function $h(G)$ defined in $\S 2.2$. Raising $G^{*}$ has the effect of violating inequality (3.15) which comes from the comparison principle approach.

Raising $G_{i n}$ has the effect of raising $M_{G}$ and $I^{*}$ and therefore, eventually, of violating condition (3.19) which is the condition for stability generated by the Lyapunov functional approach (note that $f_{2}^{\prime}$ and $f_{4}$ are uniformly bounded). On the other hand, the conditions predict convergence to the equilibrium if $f_{1}^{\prime}\left(G^{*}\right)$ is small (comparison principle approach) or $f_{1}^{\prime}\left(C_{1} V_{g}\right)$ is sufficiently small (Lyapunov functional approach). This implies that there will be no oscillations if insulin production (stimulated by glucose) is low.

\section{Appendix A}

The model proposed by Sturis et al. [19] is

$$
\begin{aligned}
\frac{d I_{p}}{d t} & =f_{1}(G)-E\left(\frac{I_{p}}{V_{p}}-\frac{I_{i}}{V_{i}}\right)-\frac{I_{p}}{t_{p}}, \\
\frac{d I_{i}}{d t} & =E\left(\frac{I_{p}}{V_{p}}-\frac{I_{i}}{V_{i}}\right)-\frac{I_{i}}{t_{i}}, \\
\frac{d G}{d t} & =G_{i n}-f_{2}(G)-f_{3}(G) f_{4}\left(I_{i}\right)+f_{5}\left(x_{3}\right), \\
\frac{d x_{1}}{d t} & =\frac{3}{t_{d}}\left(I_{p}-x_{1}\right), \\
\frac{d x_{2}}{d t} & =\frac{3}{t_{d}}\left(x_{1}-x_{2}\right), \\
\frac{d x_{3}}{d t} & =\frac{3}{t_{d}}\left(x_{2}-x_{3}\right) .
\end{aligned}
$$


In this system, $I_{p}, I_{i}$ and $G$ represent the quantities of plasma insulin (mU), intercellular insulin $(\mathrm{mU})$ and glucose $(\mathrm{mg})$ respectively. The equations are written in terms of the total amounts of these quantities. All the parameters and functional relations in the model are based on the results of independent experiments. Appropriate values for the parameters can be found in Tolic et al. [20].

The model contains three separate compartments: glucose in the plasma and intercellular space, insulin in the intercellular space and insulin in the plasma. It can be regarded as having two time delays. The time lag between the appearance of insulin in the plasma and its inhibitory effect on hepatic glucose production (see Bradley et al. [2]) is modelled as the three-stage linear filter $\left(x_{1}, x_{2}, x_{3}\right)$ and is measured by $t_{d}$. Additionally, there is a delay related to the fact that the physiological action of insulin on the utilization of glucose is regulated by the intercellular insulin rather than the plasma insulin [12], whereas glucose has a direct effect on plasma insulin. Mathematically, one could solve the second equation for $I_{i}$ in terms of $I_{p}$ and the first equation would then take the form of a distributed delay equation.

The first equation represents insulin being secreted by the pancreas into the plasma, where it is either degraded by the kidneys/liver or transported into the intercellular space. $V_{p}$ is the distribution volume for insulin in plasma and $V_{i}$ is the effective volume of the intercellular space. Insulin exchange between the two compartments is a linear function of the concentration difference between the compartments $\left(\frac{I_{p}}{V_{p}}-\frac{I_{i}}{V_{i}}\right)$ with rate constant $E$. In addition, there is linear removal of insulin from the plasma by the kidneys and the liver, with rate constant $\frac{1}{t_{p}}$. Pancreatic insulin production controlled by glucose is described by

$$
f_{1}(G)=\frac{R_{m}}{1+\exp \left(\frac{1}{a_{1}}\left(C_{1}-\frac{G}{V_{g}}\right)\right)}
$$

which has been fitted to experimental results ([11] \& [17]). The second equation of model (4.1) represents the accumulation of intercellular insulin via exchange with the plasma compartment and its degradation in muscle and adipose tissue at a rate $\frac{1}{t_{i}}$. The third equation models glucose being supplied to the plasma at an exogenously (uptake from food or intravenous glucose infusion) controlled rate $G_{\text {in }}$. The influence of insulin on hepatic glucose production, as determined by Rizza et al. [16] is described by

$$
f_{5}(I)=\frac{R_{g}}{1+\exp \left(\alpha\left(\frac{I}{V_{i}}-C_{5}\right)\right)} .
$$

Glucose utilization is represented by two terms: $f_{2}(G)$ which describes insulin-independent utilization (glucose uptake by the brain and nerve cells) and $f_{3}(G) f_{4}\left(I_{i}\right)$ which describes insulin-dependent glucose utilization (glucose uptake by muscle and fat cells). These functions are given by

$$
\begin{gathered}
f_{2}(G)=U_{b}\left(1-\exp \left(\frac{-G}{C_{2} V_{g}}\right)\right), \\
f_{3}(G)=\frac{G}{C_{3} V_{g}}
\end{gathered}
$$




$$
f_{4}(I)=U_{0}+\frac{U_{m}-U_{0}}{1+\left(\frac{I}{C_{4}}\left(\frac{1}{V_{i}}+\frac{1}{E t_{i}}\right)\right)^{-\beta}}, \quad \beta>1 .
$$

The functions (4.4), (4.5) and (4.6) are all determined by experimental data. (See Rizza et al. [16] and Verdonk et al. [21]).

\section{Appendix B}

Throughout the paper, the functions $f_{1}, f_{2}, f_{4}$ and $f_{5}$ satisfy:

$$
f_{1}(G)>0 \quad \forall G>0, f_{1}^{\prime}(G)>0 \quad \forall \quad G>0, f_{1}(0)>0 \text { and } f_{1}(G) \rightarrow a \text { as } G \rightarrow \infty,
$$

where $a>0$ is constant;

$$
f_{2}(G)>0 \quad \forall G>0, f_{2}^{\prime}(G)>0 \quad \forall \quad G>0, f_{2}(0)=0 \text { and } f_{2}(G) \rightarrow b \text { as } G \rightarrow \infty \text {, }
$$

where $b>0$ is constant;

$$
f_{4}(I)>0 \quad \forall \quad I>0, f_{4}^{\prime}(I)>0 \quad \forall \quad I>0, f_{4}(I) \rightarrow d \text { as } I \rightarrow 0,
$$

where $d>0$ is constant, and $f_{4}(I) \rightarrow e$ as $I \rightarrow \infty$

where $e>0$ is constant;

$$
f_{5}(I)>0 \quad \forall \quad I>0, f_{5}^{\prime}(I)<0 \quad \forall \quad I>0, f_{5}(0)>0 \text { and } f_{5}(I) \rightarrow 0 \text { as } I \rightarrow \infty .
$$

\section{References}

[1] Bolie, V. W. (1960) Coefficients of normal blood glucose regulation. J. Appl. Physiol. 16, 783-788.

[2] Bradley, D. C., Poulin, R. A. \& Bergman, R. N. (1993) Dynamics of hepatic and peripheral insulin effects suggest common rate-limiting step in vivo. Diabetes, 42, 296-306.

[3] Engelborghs, K., Lemaire, V., Bélair, J. \& Roose, D. (2001) Numerical bifurcation analysis of delay differential equations arising from physiological modeling. J. Math. Biol. 42, 361-385.

[4] Gourley, S. A. \& Britton, N. F. (1993) On a modified Volterra population equation with diffusion. Nonlinear Analysis, Theory, Methods \& Applications, 21, 389-395.

[5] Hagander, P., Tranberg, K. G., Thorell, J. \& Distefano, J. (1978) Models for the insulin response to intravenous glucose. Math. Biosci. 42, 15-29.

[6] Hansen, B. C., Jen, K. C., Pek, S. B. \& Wolfe, R. A. (1982) Rapid oscillations in plasma insulin, glucagon and glucose in obese and normal weight humans. J. Clin. Endocrinol. Metab. 54, 785-792.

[7] Keener, J. \& SNeyd, J. (1998) Mathematical Physiology, pp. 594-603. Springer.

[8] Landahl, H. D. \& Grodsky, G. M. (1982) Comparison of models of insulin release. Bull. Math. Biol. 44, 399-409.

[9] Lang, D. A., Matthews, D. R., Peto, J. \& Turner, R. C. (1979) Cyclic oscillations of basal plasma glucose and insulin concentrations in human beings. N. Engl. J. Med. 301, 1023-1027.

[10] LI, J., KuAng, Y. \& LI, B. (2001) Analysis of IVGTT glucose-insulin interaction models with time delay. Discrete Contin. Dyn. Syst. Ser. B, 1, 103-124.

[11] Polonsky, K. S., Given, B. D. \& van Cauter, E. (1988) Twenty-four-hour profiles and pulsatile patterns of insulin secretion in normal and obese subjects. J. Clin. Invest. 81, 442-448.

[12] Poulin, R. A., Steil, G. M., Moore, D. M., Ader, M. \& Bergman, R. N. (1994) Dynamics of glucose production and uptake are more closely related to insulin in hindlimb lymph than in thoracic duct lymph. Diabetes, 43, 180-190. 
[13] Prager, R., Wallace, P. \& Olefsky, J. M. (1986) In vivo kinetics of insulin action on peripheral glucose disposal and hepatic glucose output in normal and obese subjects. J. Clin. Invest. 78, 472-481.

[14] Redlinger, R. (1984) Existence theorems for semilinear parabolic systems with functionals. Nonlinear Anal. 8, 667-682.

[15] Redlinger, R. (1985) On Volterra's population equation with diffusion. SIAM J. Math. Anal. 16, 135-142.

[16] Rizza, R. A., Mandarino, L. J. \& Gerich, J. E. (1981) Dose-response characteristics for effects of insulin on production and utilization of glucose in man. Am. J. Physiol. 240, E630-E639.

[17] Shapiro, E. T., Tillil, H., Polonsky, K. S., Fang, V. S., Rubenstein, A. H. \& van Cauter, E. (1988) Oscillations in insulin secretion during constant glucose infusion in normal man: relationship to changes in plasma glucose. J. Clin. Endocrinol. Metab. 67, 307-314.

[18] Simon, C., Brandenberger, G. \& Follenius, M. (1987) Ultradian oscillations of plasma glucose, insulin, and C-peptide in man during continuous enteral nutrition. J. Clin. Endocrinol. Metab. 64, 669-674.

[19] Sturis, J., Polonsky, K. S., Mosekilde, E. \& van Cauter, E. (1991) Computer model for mechanisms underlying ultradian oscillations of insulin and glucose. Am. J. Physiol. 260, 439-445.

[20] Tolic, I. M., Mosekilde, E. \& Sturis, J. (2000) Modeling the insulin-glucose feedback system: the significance of pulsatile insulin secretion. J. Theor. Biol. 207, 361-375.

[21] Verdonk, C. A., Rizza, R. A. \& Gerich, J. E. (1981) Effects of plasma glucose concentration on glucose utilization and glucose clearance in normal man. Diabetes, 30, 535-537. 